\title{
A Redshift for the Intermediate Mass Black Hole Candidate HLX-1: Confirmation of its Association with the Galaxy ESO 243-49
}

\author{
K. Wiersema ${ }^{1}$, S. A. Farrell ${ }^{1}$, N. A. Webb $^{2,3}$, M. Servillat ${ }^{4}$, T. J. Maccarone ${ }^{5}$, D. Barret ${ }^{2,3}$ \\ and O. Godet ${ }^{2,3}$ \\ 1 Department of Physics and Astronomy, University of Leicester, University Road, \\ Leicester, LE1 TRH, UK \\ 2 Université de Toulouse, UPS, CESR, 9 Avenue du Colonel Roche, F-31028 Toulouse \\ cedex 9, France \\ ${ }^{3}$ CNRS, UMR5187, F-31028 Toulouse, France \\ ${ }^{4}$ Harvard-Smithsonian Center for Astrophysics, 60 Garden Street, MS-67, Cambridge, MA \\ 02138, USA \\ 5 School of Physics and Astronomy, University of Southampton, Hampshire, SO17 1BJ, UK
}

\begin{abstract}
In this Letter we report a spectroscopic confirmation of the association of HLX-1, the brightest ultra-luminous X-ray source, with the galaxy ESO 24349. At the host galaxy distance of $95 \mathrm{Mpc}$, the maximum observed $0.2-10$ $\mathrm{keV}$ luminosity is $1.2 \times 10^{42} \mathrm{erg} \mathrm{s}^{-1}$. This luminosity is $\sim 400$ times above the Eddington limit for a $20 \mathrm{M}_{\odot}$ black hole, and has been interpreted as implying an accreting intermediate mass black hole with a mass in excess of $500 \mathrm{M}_{\odot}$ (assuming the luminosity is a factor of 10 above the Eddington value). However, a number of other ultra-luminous X-ray sources have been later identified as background active galaxies or foreground sources. It has recently been claimed that HLX-1 could be a quiescent neutron star X-ray binary at a Galactic distance of only $2.5 \mathrm{kpc}$, so a definitive association with the host galaxy is crucial in order to confirm the nature of the object. Here we report the detection of the $\mathrm{H} \alpha$ emission line for the recently identified optical counterpart at a redshift consistent with that of ESO 243-49. This finding definitively places HLX-1 inside ESO 243-49, confirming the extreme maximum luminosity and strengthening the case for it containing an accreting intermediate mass black hole of more than $500 \mathrm{M}_{\odot}$.
\end{abstract}

Subject headings: accretion, accretion disks - X-rays: binaries - X-rays: individual (ESO 243-49 HLX-1) 


\section{Introduction}

Ultra-luminous X-ray (ULX) sources are extragalactic objects located outside the nucleus of the host galaxy with bolometric luminosities $>2.6 \times 10^{39} \mathrm{erg} \mathrm{s}^{-1}$ (e.g. Roberts 2007). These extreme luminosities appear to exceed the theoretical Eddington limit for a stellar mass black hole, where the gravitational pressure of in-falling material balances against the radiation pressure, implying the presence of accreting black holes with masses between $\sim 10^{2}$ $-10^{5} \mathrm{M}_{\odot}$. The existence of such intermediate mass black holes is widely disputed, as hyperaccretion (Begelman 2002) and/or beaming (Körding et al. 2002; Freeland et al. 2006; King 2008) can cause a stellar mass black hole to appear to exceed the Eddington limit. However, the rare sub-class of hyper-ULXs with luminosities $>10^{41} \mathrm{erg} \mathrm{s}^{-1}$ require extreme tuning to explain without the presence of an intermediate mass black hole (e.g Roberts 2007; Miller 2009), needing either non-physical levels of super-Eddington accretion or very small beaming factors. The luminosity derived for these objects is entirely dependent upon the X-ray source being located within the host galaxy so ruling out background (e.g. Clark et al. 2005) or foreground (e.g. Weisskopf 2004) sources is essential. This is normally not possible to achieve with the X-ray data alone, and so multi-wavelength follow-up observations are required in order to confirm the ULX nature. For example, the previous title-holder for the brightest ULX in the galaxy MCG-03-34-63 (Miniutti et al. 2006) has recently been found to be a background Active Galactic Nucleus (AGN; Miniutti et al. in preparation).

The brightest ULX candidate currently known is the X-ray source HLX-1 coincident with the galaxy ESO 243-49, with a derived maximum luminosity (assuming it is at the galaxy redshift of $\mathrm{z}=0.0224$ ) of $\sim 10^{42} \mathrm{erg} \mathrm{s}^{-1}$ (Farrell et al. 2009). This object is almost an order of magnitude more luminous than the other hyper-ULXs (e.g. Gao et al. 2003), and would provide strong evidence for the existence of intermediate mass black holes if the luminosity can be confirmed. Following its discovery, HLX-1 has been regularly monitored in X-rays by the Swift observatory, and has been found to vary by a factor of $\sim 20$ over relatively short time scales, at the same time exhibiting spectral variability similar to the canonical

state transitions seen in Galactic stellar mass black hole binaries (Godet et al. 2009). The X-ray source position was recently improved to sub-arcsecond accuracy using data from the Chandra space telescope (Webb et al. 2010), allowing a faint optical counterpart $(\mathrm{R}=$ $23.8 \mathrm{mag}$ ) to be identified (Soria et al. 2010). The maximum X-ray to optical flux ratio $\left(\mathrm{F}_{x} / \mathrm{F}_{\text {opt }}\right)$ of HLX-1 is $~ 1000$, two orders of magnitude higher than is typically seen for AGN (e.g Severgnini et al. 2003), making a background source highly unlikely. However, it has recently been argued that the X-ray spectrum and $\mathrm{F}_{x} / \mathrm{F}_{\text {opt }}$ ratio of HLX-1 were consistent with a Galactic quiescent neutron star X-ray binary with a late-type secondary at a distance of $\sim 2.5 \mathrm{kpc}$ (Soria et al. 2010). These objects contain neutron stars accreting at low rates from a low-mass companion star, and have high $\mathrm{F}_{x} / \mathrm{F}_{\text {opt }}$ ratios and $\mathrm{X}$-ray spectra that are 
typically dominated by thermal emission from the surface or atmosphere of the neutron star (e.g. Webb \& Barret 2007; Jonker 2008).

In this Letter we present spectroscopic observations of HLX-1 with the Very Large Telescope (VLT). In $\S 2$ we describe the observations and in $\S 3$ we outline the data reduction and analysis steps. $\S 4$ reports the results that we obtained, and $\S 5$ presents a discussion and the conclusions that we have drawn.

\section{Observations}

Following the detection of the counterpart we obtained optical spectroscopic data using the VLT accorded under the Directors Discretionary Time (DDT) program (ID: 284.D5008). The VLT acquisition absolute astrometry is not good enough to acquire blindly a given position in RA and Dec when the source is not detectable by the acquisition camera, as the typical uncertainties exceed the slit width. A commonly used method is to use blind offsets from the position of a bright reference star. The offset star is acquired accurately using a through-the-slit exposure after which the blind offset is performed. The typical uncertainty associated with this method is $\sim 0.3^{\prime \prime}$ or better. However, the most accurate method is to use fortuitous alignment of foreground stars with the target. With long slits such as that of FORS2, the chances of two random bright objects aligning with the target is fairly large, provided that there are no strict requirements for the slit position angle or parallactic angle. To find such alignments, we used a deep image of the field in the J band (theoretical detection limit of $\mathrm{J}=22.5 \mathrm{mag}$ ) that we obtained with PANIC at the Baade Magellan Telescope on 2009 August 8. We found two field stars of J $\sim 20$ mag that allowed a precise positioning of the long slit of FORS2 so that it would cover the position of HLX-1 (see Figure 1). The two stars can be centered in the slit very accurately (to the accuracy of the centroid determination of each star, so considerably better than 1 pixel).

We obtained deep spectroscopy on four nights, using the FORS2 instrument on Unit Telescope 1 (UT1) of the VLT. A 1.0" wide slit was used with a fixed position angle aligned on two field stars such that it would cover the position of HLX-1 (Figure 1). The Chandra position of HLX-1 has an offset of $0.14^{\prime \prime}$ from the center of the slit, while the R-band counterpart detected by Soria et al. (2010) has an offset of $0.006^{\prime \prime}$ from the center of the slit. As the error on both the Chandra and R-band positions are $0.3^{\prime \prime}$, both positions are well within the slit. This slit angle cuts through the bulge of the galaxy, missing the brightest part of the nucleus. Seeing conditions during the observations (as measured from the acquisition data) were good on the December observing dates $\left(0.7-0.9^{\prime \prime}\right)$, but were considerably worse on the November observing date $\left(1.1-1.5^{\prime \prime}\right)$. 
A log of the spectroscopic observations can be found in Table 1, To maximize the wavelength range in the red part of the optical spectrum we used the 300I grism, with the OG590 filter used to suppress contamination by the second spectral order. This results in a nominal wavelength range $\sim 5860 \AA$ to $1.2 \mu \mathrm{m}$, though the signal redwards of $10500 \AA$ is too weak to be useful.

\section{Data Reduction \& Analysis}

The data were reduced by using the standard procedures in IRAF1 within the ccdred and twodspec packages, using bias frames and flat-fields taken the same night. The individual reduced $600 \mathrm{~s}$ spectra from the December observations were co-added, with the cosmic rays removed in the process. The data from 26 November 2009 were excluded from the summed spectrum, as the poor seeing on that night meant that the inclusion of those data did not increase the overall signal-to-noise of the data set.

We extracted the spectra from the merged 2-D spectrum (Figure 21) by using the relatively bright continuum of the bulge to fit the shape of the trace function, and extracted 66 adjoining, equally sized sub-apertures along this trace. The pixel scale in the spatial direction of Figure 2 is $0.25^{\prime \prime}$ pixel $^{-1}$. The sub-apertures were $0.9^{\prime \prime}$ wide, giving a width slightly larger than the seeing full width at half maximum (FWHM) of the combined frame. At $\mathrm{z}=0.0224$ this corresponds to a physical scale of $\sim 0.4 \mathrm{kpc}$ per sub-aperture, assuming a cosmology $\mathrm{H}_{0}=71 \mathrm{~km} \mathrm{~s}^{-1} \mathrm{Mpc}^{-1}, \Omega_{m}=0.27, \Omega_{\Lambda}=0.73$. The series of sub-apertures chosen encompasses everything in between (and including) the two slit alignment stars. Furthermore, the position of the aperture and its subdivision into sub-apertures was chosen such that the expected position of the trace of HLX-1, determined through relative offsets with

\footnotetext{
${ }^{1}$ http://www.iraf.net/
}

Table 1: VLT FORS2 Observation Log.

\begin{tabular}{cc}
\hline $\begin{array}{c}\text { Start Date } \\
(\mathrm{UT})\end{array}$ & $\begin{array}{c}\text { Exposure Time } \\
(\mathrm{s})\end{array}$ \\
\hline $26 / 11 / 200902: 57$ & $4 \times 600$ \\
$05 / 12 / 200901: 11$ & $4 \times 600$ \\
$06 / 12 / 200900: 51$ & $4 \times 600$ \\
$07 / 12 / 200901: 00$ & $4 \times 600$ \\
\hline
\end{tabular}


respect to the two slit alignment stars, was centred within one sub-aperture.

In the following we will refer to the spectra extracted with these small apertures as sub-spectra. The sub-spectra were wavelength calibrated using $\mathrm{He}, \mathrm{HgCd}$ and $\mathrm{Ar}$ arc lamp spectra. From a velocity cross-correlation of the wavelength calibrated arc spectrum with itself we measured a spectral resolution of $550 \mathrm{~km} \mathrm{~s}^{-1}$ (FWHM). We boxcar smoothed the 2-D spectrum in the spatial direction with a 9 pixel box size, and subtracted this resulting image from the original 2-D spectrum to perform a crude subtraction of the bright galaxy profile. In this manner we subtracted the galaxy $\mathrm{H} \alpha$ absorption line (likely stellar in origin), while not introducing artefacts due to the galaxy rotation curve (for which the scale is much larger than 9 pixels).

\section{Results}

The galaxy bulge sub-spectra show strong absorption bands typical of a S0 type galaxy. The Na ID absorption feature can be seen in the blue end of the spectrum, giving a redshift for the galaxy of $\mathrm{z}=0.0223$ consistent with previous measurements (Afonso et al. 2005). The $\mathrm{H} \alpha$ line in absorption is also clearly detected at the same redshift, with an obvious curve in the 2-D spectrum due to the rotation of the galaxy spiral arms (Figure 21). The absorption line visible at $6643 \AA$ is the rest-frame $6497 \AA$ blend (BaII, FeI and CaI) well known in late- $\mathrm{G}$ and early-K stars, which probably dominates in this galaxy and is consistent with the $\sim 5$ Gyr old stellar population derived by Soria et al. (2010). In addition to these features, a faint trace can be seen in the 2-D spectrum at the position of HLX-1, despite its proximity to the bright galaxy nucleus. The $\mathrm{H} \alpha$ absorption feature is also present in the HLX-1 trace, with visual inspection finding this feature to be partially filled in.

To confirm the association, we extracted sub-spectra from the position of HLX-1 and from four sub-apertures taken from neighboring regions either side of the HLX-1 position in the slit. The fluxes of the neighboring sub-spectra were interpolated in count space as a function of wavelength to the HLX-1 sub-spectrum using a spline function in order to estimate the background at the source position (Figure 3), and then subtracted from the source sub-spectrum. The resulting background subtracted spectrum reveals an emission line with an approximately Gaussian profile superimposed on a very weak continuum (Figure 4). The width of the spectral bins in both Figures 3 and 4 is $3.2 \AA$. There is no other evidence of $\mathrm{H} \alpha$ in emission anywhere else across the galaxy, despite the fact that the slit runs across part of the UV-bright region of ESO 243-49 in the low-resolution GALEX and Swift UVOT images (see Webb et al. 2010). This could indicate that star formation is uniform in this part of the galaxy, so that any $\mathrm{H} \alpha$ signatures are subtracted out in the residual image, or that the 
emission features are too faint. However, without knowing the structure and scale of the UV emission we cannot be certain that the UV emission region falls in the slit. Higher resolution UV imaging with the Hubble Space Telescope in cycle 18 has recently been awarded us, so we should be able to answer this question in the near future.

To quantify the line significance, flux and wavelength, we used the sub-spectra. We performed a subtraction of the bright galaxy light from the HLX-1 sub-spectrum by using the interpolated background spectrum described above. Flux calibration was performed on the resulting galaxy subtracted HLX-1 spectrum using observations of the white dwarf standard star BPM 16274. We note that there are likely fairly large systematic errors associated with the procedure above: the bright galaxy brightness profile, though fairly smooth, changes on spatial scales similar to the sub-spectrum aperture sizes, so weighted linear interpolation of the adjacent sub-apertures can easily over or underestimates the contribution of the galaxy to the HLX-1 sub-spectrum. These systematic errors make it very difficult to estimate the absolute flux of the continuum spectrum; however, the shape of the spectrum is not dependent on which sub-spectra were chosen for the background subtraction, or the interpolation method that was employed (e.g. spline vs linear interpolation functions). Hence, the presence of a significant emission line is not in doubt.

By fitting a Gaussian function we measure the wavelength of the emission line to be $6721.0 \pm 1.1 \AA$ and its FWHM to be $15.2 \pm 2.3 \AA$. This value is consistent with the instrumental resolution, and hence the line width should be taken as an upper-limit. The line significance was calculated from the 2-D spectrum once the smoothed spectrum was subtracted. We integrated the number of counts (ADUs) in the line in a rectangular aperture centered on the centroid of the Gaussian fit with a size twice the line FWHM in the wavelength direction and twice the seeing FWHM in the spatial direction. The error was calculated from the error in the resulting integrated counts and the standard deviation of the local background (i.e. the residuals from the subtraction). Thus the integrated significance of the $\mathrm{H} \alpha$ line, taking into account the error introduced by the subtraction procedure, was calculated to be $11.3 \sigma$. The flux of the emission line is estimated to be $1.6 \times 10^{-17} \mathrm{erg} \mathrm{s}^{-1}$ $\mathrm{cm}^{-2}$. However, as the galaxy subtraction procedure introduces large systematic uncertainties, we adopt a conservative order of magnitude estimate for the line flux of $\sim 10^{-17} \mathrm{erg} \mathrm{s}^{-1}$ $\mathrm{cm}^{-2}$. The flux of the continuum cannot be reliably measured with this data, as the low count rate is smaller than the errors introduced by interpolating the background. 


\section{Discussion \& Conclusions}

The wavelength of the emission line detected in the HLX-1 spectrum is consistent with $\mathrm{H} \alpha$ at the redshift of ESO 243-49, with a velocity offset of $\sim 170 \mathrm{~km} \mathrm{~s}^{-1}$ (considerably smaller than the galaxy rotation curve). The only other plausible identification of this line is [OII] for a source at $\mathrm{z}=0.80$; however, the $\mathrm{F}_{x} / \mathrm{F}_{\text {opt }}$ ratio of $\sim 1000$ argues strongly against a background AGN. We therefore conclude that the line is most likely $\mathrm{H} \alpha$ emission from an object gravitationally bound to ESO 243-49.

A method for estimating the mass of black holes in low-redshift AGN has been established solely using observations of the $\mathrm{H} \alpha$ emission line (Greene \& Ho 2005). A self-consistent picture could thus be developed in which the line width-luminosity-mass relation seen for AGN can be extrapolated down to lower black hole masses. At the galaxy redshift the line luminosity is $\sim 2 \times 10^{37} \mathrm{erg} \mathrm{s}^{-1}$, so if we assume that the $\mathrm{H} \alpha$ scaling relationship holds for lighter black holes, the line luminosity and FWHM imply a black hole mass of $\sim 1500 \mathrm{M}_{\odot}$ for HLX-1. However, as the line is unresolved in our spectrum, this mass estimate should be taken as an upper-limit. In addition, this relationship would only hold if the origin of the $\mathrm{H} \alpha$ emission is the same as in AGN (i.e. the broad line region). Alternatively, the $\mathrm{H} \alpha$ line could be produced by the environment in which HLX-1 lies, perhaps a star cluster or photoionized/shock-ionized gas nebula, in which case this method would not apply and the mass upper-limit would not stand. The large uncertainties inherent in the process of subtracting the diffuse emission from the galaxy mean that we are not able to set any meaningful limits on the continuum flux. Thus, with the data at hand we cannot place any new constraints on the nature of the optical counterpart (e.g. star cluster, globular cluster, nucleated dwarf galaxy etc.), beyond what can be done using the photometry already reported in Soria et al. (2010).

The detection of a $\mathrm{H} \alpha$ emission line at a redshift of $\mathrm{z}=0.0223$ definitively places HLX1 within ESO 243-49, confirming the $10^{42} \mathrm{erg} \mathrm{s}^{-1}$ luminosity. As such HLX-1 continues to provide the strongest case to date for the existence of intermediate mass black holes in at least some ULXs. The large-scale temporal and spectral variability of this object therefore provides us with a unique opportunity to study an intermediate mass black hole in different accretion regimes, allowing us to make direct comparisons with the populations of stellar mass and super-massive black holes. An ongoing monitoring program in X-rays with the Swift observatory and planned follow-up observations at other wavelengths should continue to shed light on the nature and environment of this enigmatic object.

We thank M. C. Miller and the anonymous referees for their comments that helped to improve this paper. We thank Tim de Zeeuw for according us the VLT DDT observa- 
tions. Based on observations made with ESO Telescopes at the Paranal Observatory under programme ID 284.D-5008. IRAF is distributed by the National Optical Astronomy Observatories, which are operated by the Association of Universities for Research in Astronomy, Inc., under cooperative agreement with the National Science Foundation. S.A.F., K.W. and T.J.M. acknowledge STFC funding. T.J.M. thanks the European Union FP7 for support through grant 215212 Black Hole Universe. M.S. is supported in part by Chandra grants AR9-0013X and GO9-0102X.

Facilities: VLT (FORS2).

\section{REFERENCES}

Afonso, J., Georgakakis, A., Almeida, C., Hopkins, A. M., Cram, L. E., Mobasher, B., \& Sullivan, M. 2005, ApJ, 624, 135

Begelman, M. C. 2002, ApJ, 568, L97

Clark, D. M., et al. 2005, ApJ, 631, L109

Farrell, S. A., Webb, N. A., Barret, D., Godet, O., \& Rodrigues, J. M. 2009, Nature, 460, 73

Freeland, M., Kuncic, Z., Soria, R., \& Bicknell, G. V. 2006, MNRAS, 372, 630

Gao, Y., Wang, D. Q., Appleton, P. N., \& Lucas, R. A. 2003, ApJ, 596, L171

Godet, O., Barret, D., Webb, N. A., Farrell, S. A., \& Gehrels, N. 2009, ApJ, 705, L109

Greene, J. E., \& Ho, L. 2005, ApJ, 630, 122

Jonker, P. G. 2008, 40 Years of Pulsars: Millisecond Pulsars, Magnetars and More, 983, 519

King, A. R. 2008, MNRAS, 385, L113

Körding, E., Falcke, H., \& Markoff, S. 2002, A\&A, 382, L13

Miller, M. C. 2009, Nature Physics, 5, 537

Miniutti, G., Ponti, G., Dadina, M., Cappi, M., Malaguti, G., Fabian, A. C., \& Gandhi, P. 2006, MNRAS, 373, L1

Roberts, T. P. 2007, Ap\&SS, 311, 203

Severgnini, P., et al. 2003, A\&A, 406, 483 
Soria, R., Hau, G. K. T., Graham, A. W., Kong, A. K. H., Kuin, N. P. M., Li, I.-H., Liu, J.-F., \& Wu, K. 2010, MNRAS, 405, 870

Webb, N. A., \& Barret, D. 2007, ApJ, 671, 727

Webb, N. A., Barret, D., Godet, O., Servillat, M., Farrell, S. A., \& Oates, S. R. 2010, ApJ, 712, L107

Weisskopf, M. C., Wu, K., Tennant, A. F., Swartz, D. A., \& Ghosh, K. K. 2004, ApJ, 605, 360 


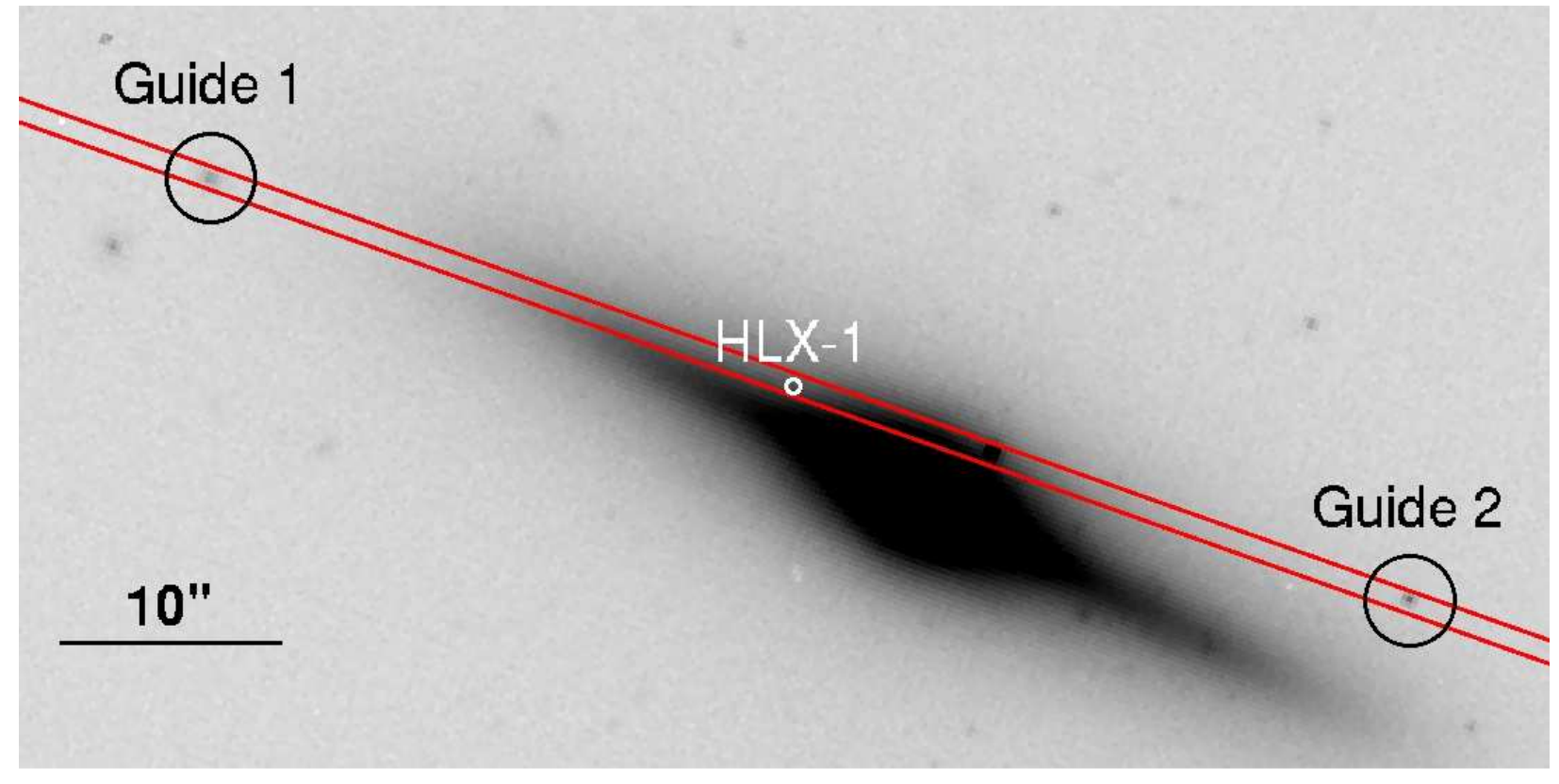

Fig. 1.- I-band VLT optical image of ESO 243-49. The slit position (red box) with a width of $1^{\prime \prime}$ was aligned on two guide stars that fortuitously lined up with the Chandra position of HLX-1 (white circle, with the radius corresponding to the $0.3^{\prime \prime}$ positional error). 


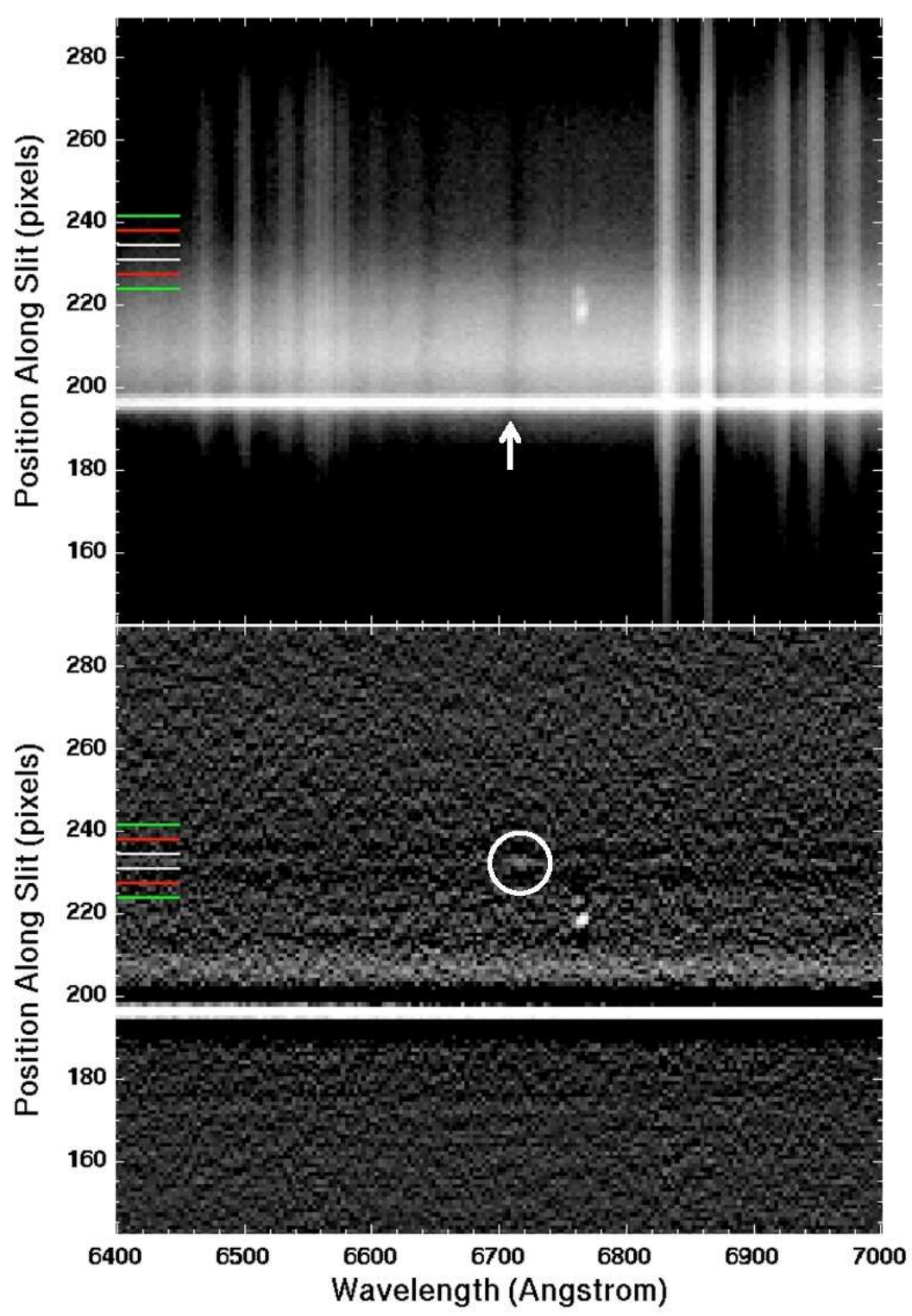

Fig. 2.- Top: the VLT 2-D spectrum of the HLX-1 field, with the ESO 243-49 H $\alpha$ absorption line indicated by the white arrow. Bottom: 2-D spectrum after the diffuse galaxy emission has been removed via subtraction of a smoothed image. The circle indicates the position of the $\mathrm{H} \alpha$ emission line in the HLX-1 spectrum. The nearby emission line source $\left(\sim 3^{\prime \prime}\right.$ away from HLX-1, so well outside the Chandra error circle) appears to be a background galaxy. The sub-aperture covering the position of HLX-1 lies between the white horizontal lines to the left of the plots. The neighboring sub-apertures used to interpolate the background spectrum lie between the red and green and red and white lines. 


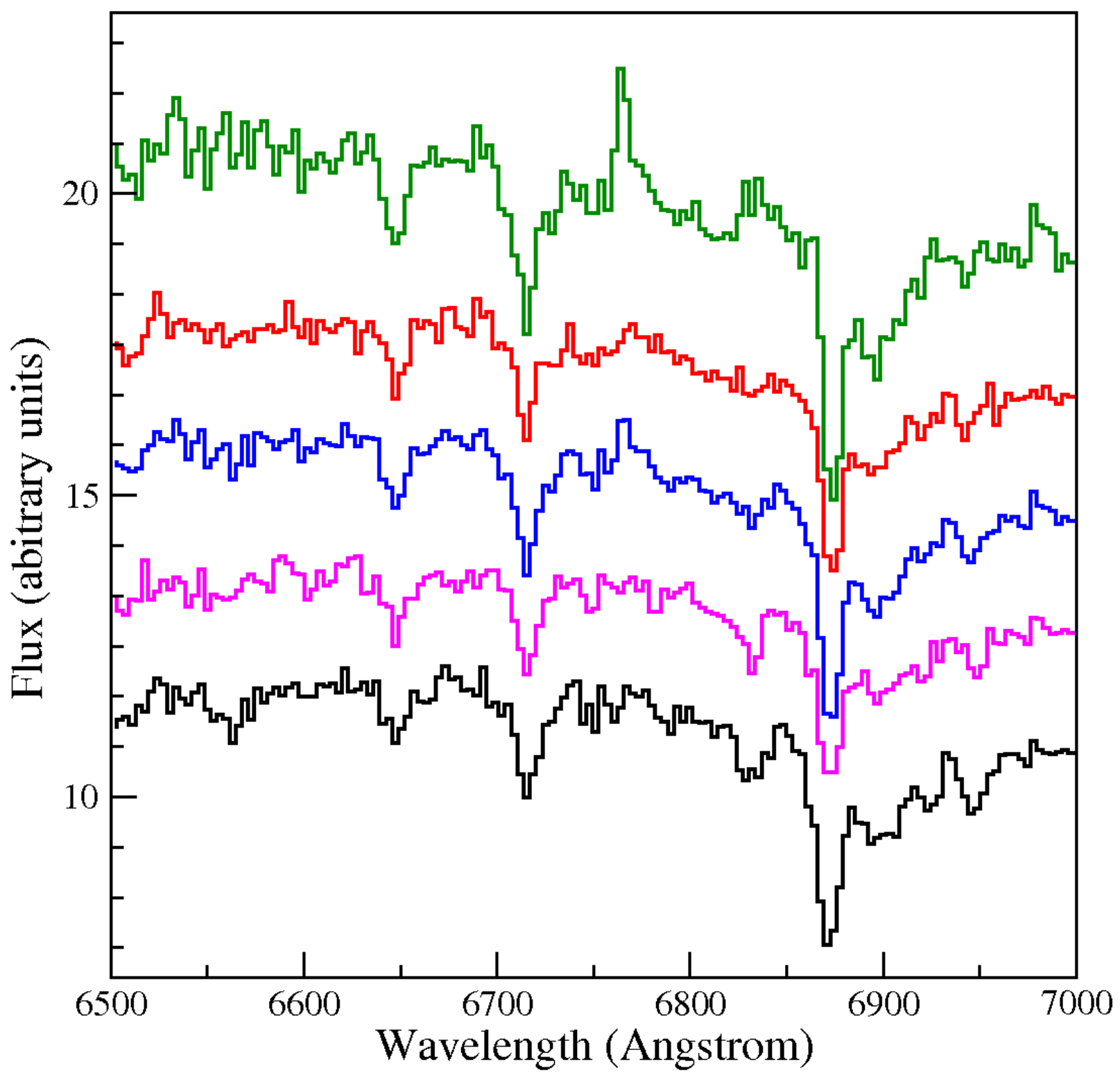

Fig. 3.- Spectra from the neighboring sub-apertures (green, red, pink and black) that were used to derive the interpolated background spectrum at the position of HLX-1 (blue). A slight offset has been included to the arbitrary fluxes for the purpose of clarity. 


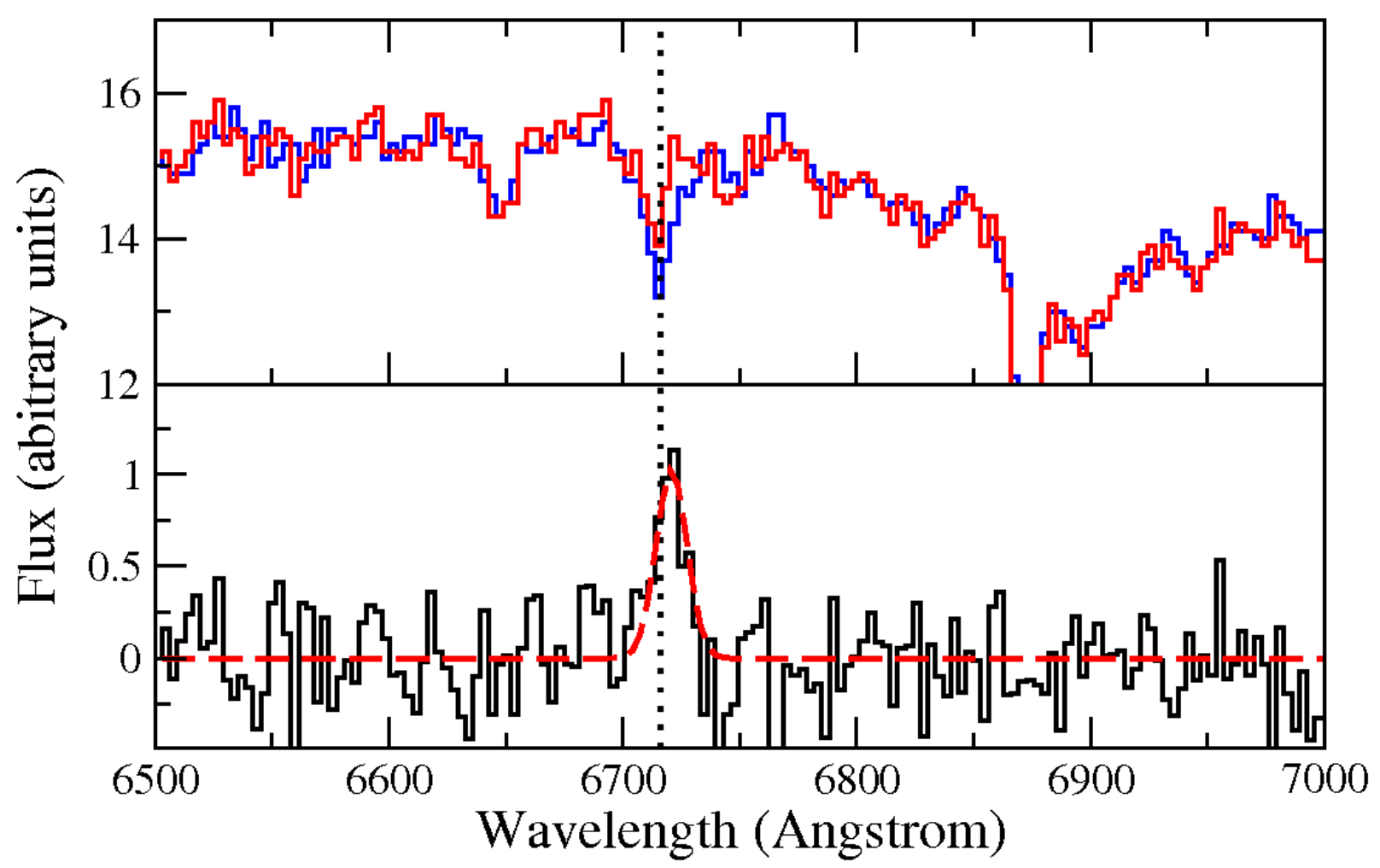

Fig. 4.- Top: 1-D spectrum extracted from the position of HLX-1 (red) and the background spectrum (blue) interpolated from neighbouring sub-apertures. The absorption line at 6716 $\AA$ is $\mathrm{H} \alpha$ at the redshift of ESO 243-49. The signal-to-noise ratio around this absorption feature is $\sim 40$ pixel $^{-1}$ in the HLX-1 sub-spectrum. Bottom: background subtracted spectrum of HLX-1, showing line emission at $6721 \AA$ with a significance of $11.3 \sigma$. The red dashed line indicates the Gaussian model fitted to the emission line. The dotted vertical line indicates the wavelength of the $\mathrm{H} \alpha$ absorption line in the ESO 243-49 spectrum. In both plots the y-axis indicates arbitrary flux units. 